The University of San Francisco

USF Scholarship: a digital repository @ Gleeson Library |

Geschke Center

Psychology

College of Arts and Sciences

2010

\title{
Neural Activity in the Hippocampus and Perirhinal Cortex During Encoding Is Associated With the Durability of Episodic Memory
}

\author{
V.A. Carr \\ Indre Viskontas \\ University of San Francisco, ivviskontas@usfca.edu \\ S. A. Engel \\ B.J. Knowlton
}

Follow this and additional works at: http://repository.usfca.edu/psyc

Part of the Neurology Commons, and the Psychology Commons

\section{Recommended Citation}

Carr, V.A., Viskontas, I.V., Engel, S.A., Knowlton, B.J. Neural activity in the hippocampus and perirhinal cortex during encoding is associated with the durability of episodic memory (2010) Journal of Cognitive Neuroscience, 22 (11), pp. 2652-2662.

https://doi.org/10.1162/jocn.2009.21381

This Article is brought to you for free and open access by the College of Arts and Sciences at USF Scholarship: a digital repository @ Gleeson Library | Geschke Center. It has been accepted for inclusion in Psychology by an authorized administrator of USF Scholarship: a digital repository @ Gleeson Library| Geschke Center. For more information, please contact repository@usfca.edu. 


\title{
Neural Activity in the Hippocampus and Perirhinal Cortex during Encoding Is Associated with the Durability of Episodic Memory
}

\author{
Valerie A. Carr*, Indre V. Viskontas*, Stephen A. Engel, \\ and Barbara J. Knowlton
}

\begin{abstract}
Studies examining medial temporal lobe (MTL) involvement in memory formation typically assess memory performance after a single, short delay. Thus, the relationship between MTL encoding activity and memory durability over time remains poorly characterized. To explore this relationship, we scanned participants using high-resolution functional imaging of the MTL as they encoded object pairs; using the remember/know paradigm, we then assessed memory performance for studied items both 10 min and 1 week later. Encoding trials were classified as either subsequently recollected across both delays, transiently recollected (i.e., recollected at 10 min but not after 1 week), consistently familiar, or consistently forgotten. Activity in perirhinal cortex (PRC) and a hippo-
\end{abstract}

\section{INTRODUCTION}

Shortly following an event, we often have a vivid episodic memory for what transpired, yet over time, this vividness may fade or the event may be forgotten altogether. For other events our memories endure, and we are able to recollect details of the event over extended periods. What neural mechanisms might account for these differences in the durability of memory? The medial temporal lobe (MTL) is known to play a key role in both encoding and retrieving declarative memories (for a review, see, e.g., Squire, Stark, \& Clark, 2004). It remains unclear, however, how the strength of encoding within this region influences memory durability, given that factors occurring postencoding, such as memory consolidation and retrieval accessibility, also affect memory durability. Furthermore, the manner in which the hippocampal region (subiculum, CA fields, and dentate gyrus) and surrounding cortical regions (parahippocampal [PHC], perirhinal [PRC], and entorhinal [ERC] cortices) contribute to successful encoding remains controversial. Using high-resolution fMRI of the MTL to bridge these two areas of research, the current

University of California, Los Angeles

*These authors contributed equally to this work. campal subfield comprising the dentate gyrus and CA fields 2 and 3 reflected successful encoding only when items were recollected consistently across both delays. Furthermore, in PRC, encoding activity for items that later were consistently recollected was significantly greater than that for transiently recollected and consistently familiar items. Parahippocampal cortex, in contrast, showed a subsequent memory effect during encoding of items that were recollected after $10 \mathrm{~min}$, regardless of whether they also were recollected after 1 week. These data suggest that MTL subfields contribute uniquely to the formation of memories that endure over time, and highlight a role for PRC in supporting subsequent durable episodic recollection.

study aimed to evaluate the potential for functional heterogeneity among MTL subfields in supporting the formation of vivid memories that endure over time.

Findings from standard-resolution imaging studies lend support to the notion that subsequent memory success and MTL encoding activity are related, although such studies typically assess memory performance after a single, short delay (e.g., less than $24 \mathrm{hr}$ ), and thus, do not speak directly to the issue of memory durability. For example, early fMRI studies examining the relationship between encoding activity and subsequent memory success demonstrated increased encoding activity for subsequently recognized versus forgotten items in the parahippocampal gyrus (Brewer, Zhao, Desmond, Glover, \& Gabrieli, 1998; Wagner et al., 1998), as well as increased hippocampal activity for successfully versus unsuccessfully recalled words (Fernandez et al., 1998). Several theories of MTL function, however, suggest that the hippocampus and surrounding cortices differentially support encoding of new memories, although the organizing principles underlying such differences remain a topic of debate.

Building from MTL anatomy and neural network principles, several computational theories posit that MTL cortices are specialized for slowly developing representations of generalities in the environment, such that similar representations are assigned to similar stimuli. Conversely, such 
models propose that the hippocampus plays a key role in assigning distinct representations to cortical input patterns, and for binding co-occurring input patterns into cohesive event memories (Norman \& O’Reilly, 2003). Differences in the way information is represented in these structures are then thought to lead to regional differences in supporting recognition memory. Specifically, such computational theories suggest that the hippocampus encodes patterns of cortical input in a manner that supports subsequent detailed recollection of episodes, whereas MTL cortices are thought to be incapable of supporting memory for specific events due to encoding of overlapping representations, and instead enable a sense of familiarity (Norman \& O'Reilly, 2003; O’Reilly \& Rudy, 2001). Additional support for this division of labor comes from theories of MTL function based upon empirical findings in animals and humans suggesting that the hippocampus and PRC make dissociable contributions to recognition memory (Brown \& Aggleton, 2001; Aggleton \& Brown, 1999).

Support from neuroimaging studies for this hippocampal/ cortical distinction has been equivocal, however. Several recent reviews of the MTL literature suggest a common pattern of findings such that anterior portions of the parahippocampal gyrus (typically assumed to map onto PRC) support encoding of the individual elements of an event leading to subsequent feelings of familiarity, whereas the posterior extent (PHC) supports encoding of spatial information and the context in which individual elements occur, enabling later episodic recollection. The hippocampus is thought to bind together these different aspects of an experience into a cohesive event memory that later can be recollected in detail (Diana, Yonelinas, \& Ranganath, 2007; Eichenbaum, Yonelinas, \& Ranganath, 2007; Davachi, 2006). These findings support the idea that both the hippocampus and PHC are involved in encoding episodic memories, but that each region's contribution to subsequent recollection is different. Furthermore, emerging data suggest that although PRC may play a key role in item encoding, under certain conditions, this region may also support encoding of associations between items or between items and their features (Haskins, Yonelinas, Quamme, \& Ranganath, 2008; Staresina \& Davachi, 2006, 2008; Tendolkar et al., 2007), suggesting that later associative recollection may depend on PRC activity during encoding. Finally, although there is broad agreement regarding the role of the hippocampus in encoding information in a manner that enables later recollection, controversy exists regarding the degree to which this region selectively supports recollection during retrieval (e.g., Squire, Wixted, \& Clark, 2007; Squire et al., 2004).

These findings have led several researchers to conclude that drawing a sharp distinction between the hippocampus and parahippocampal gyrus in encoding information in a manner supportive of later recollection and familiarity, respectively, is an oversimplified description of MTL function (Diana et al., 2007; Eichenbaum et al., 2007; Davachi, 2006; Squire et al., 2004). Thus, although there is little doubt that MTL encoding activity positively correlates with future memory success, additional research is required to better understand the precise manner and conditions under which the hippocampus and adjacent cortical regions support encoding of memories subsequently recollected in vivid detail versus those judged merely familiar. Furthermore, the vast majority of studies attempting to elucidate the role of the hippocampus and MTL cortices in forming new memories has evaluated memory performance after a short delay, and thus, does not offer insights into MTL contributions to forming memories that endure over time.

One exception is a recent whole-brain study (Uncapher \& Rugg, 2005) in which participants first encoded a set of items while undergoing fMRI, and then were tested on half of the studied items after a 30-min delay, with the remaining half tested after a 48-hr delay. The authors found that encoding activity in the anterior hippocampus was associated with successful recollection at both the 30-min and 48-hr delay, and that activity in the parahippocampal gyrus was correlated with successful familiarity-based recognition at both delays. Although this procedure enabled the authors to examine the relationship between encoding activity and subsequent memory success at either a short or long delay, testing different sets of items at the two time points limited the authors' ability to assess how durable a given memory was over time. For example, items successfully recollected at the short delay likely included items that would have lost their associated episodic character over the 48-hr delay. Similarly, a portion of items deemed familiar at the long delay likely would have been recollected initially had they been tested.

Using a task design that enabled us to track participants' memory across a short and long delay, the current study aimed to directly address the hypothesis that MTL encoding activity relates to how durable a given memory is over time. To this end, we used high-resolution fMRI to measure patterns of MTL encoding activity as participants studied a series of object pairs. We then assessed memory performance for the same items 10 min and 1 week after the initial encoding session using the remember/know procedure (Tulving, 1985). Based upon behavioral performance, we evaluated MTL encoding activity associated with items subsequently recollected across both delays and contrasted it with activity associated with items initially recollected but later deemed familiar ("transiently recollected"), items consistently familiar, and items consistently forgotten. Furthermore, our use of high-resolution imaging of the MTL allowed for precise localization of subfields within the hippocampus and parahippocampal gyrus, which in turn, enabled us to evaluate the potential for functional heterogeneity among subfields in supporting vivid, durable memories.

Taking the view that the unique anatomy and connectivity of the hippocampus is specialized to support episodic encoding, we hypothesized that the hippocampus would be most active during encoding of consistently recollected 
memories. Thus, consistently recollected items should be associated with greater hippocampal activity during encoding than those that are transiently recollected, consistently familiar, or consistently forgotten. Regarding MTL cortical activity, two alternate predictions were made. Several computational models posit that the MTL cortices do not specifically contribute to episodic encoding; thus, activity across conditions should not differ with the exception of a general subsequent memory effect (greater activity for subsequently successful than unsuccessful recognition, regardless of whether the memory was episodic). In contrast, theories of MTL function based upon findings in the neuroimaging literature suggest that PHC is involved in encoding of spatial or contextual information that supports subsequent episodic recollection. Given that our stimuli are not obviously spatial in nature, it was unclear to what degree they would engage PHC; however, theories regarding a broader role of this region in processing contextual information suggest that it might exhibit a similar pattern of activity as that in the hippocampus. Finally, although PRC is widely thought to encode item information supportive of subsequent feelings of familiarity, emerging data suggest that this region may engage in encoding certain forms of associations capable of supporting later recollection. For this reason, we did not make strong hypotheses regarding the relationship between PRC encoding activity and the subsequent durability of episodic memory.

\section{EXPERIMENTAL METHODS}

\section{Participants}

Twelve healthy individuals (6 women), of whom all but one were right-handed, participated in this study. The inclusion of the left-handed participant's data did not change the overall pattern of results, thus data from all 12 participants are described herein. Participants were fluent English speakers and between 25 and 30 years of age (mean = $27.9 \pm 2.15$ ). Each participant was paid $\$ 100$ for participation in this study. The study was performed under a protocol approved by the UCLA Office for Protection of Research Participants.

\section{Materials}

At encoding, participants viewed 150 object pairs (Snodgrass \& Vanderwart, 1980) with associated object names printed below the pictures. Pairs were presented in two orientationshorizontally or vertically - and the first or "cue" item of each pair was presented in one of four colors: blue, yellow, pink, or green. The second object was always presented in black and white (see Figure 1). For counterbalancing purposes, each orientation and color appeared with approximately the same frequency; additionally, in order to counterbalance stimuli used for cues and lures, we assigned each participant to one of two groups. One-third of the object pairs were unique to
Figure 1. Encoding paradigm. Object pairs differed in color and orientation on the screen, and participants were instructed to imagine items within a pair interacting with one another.

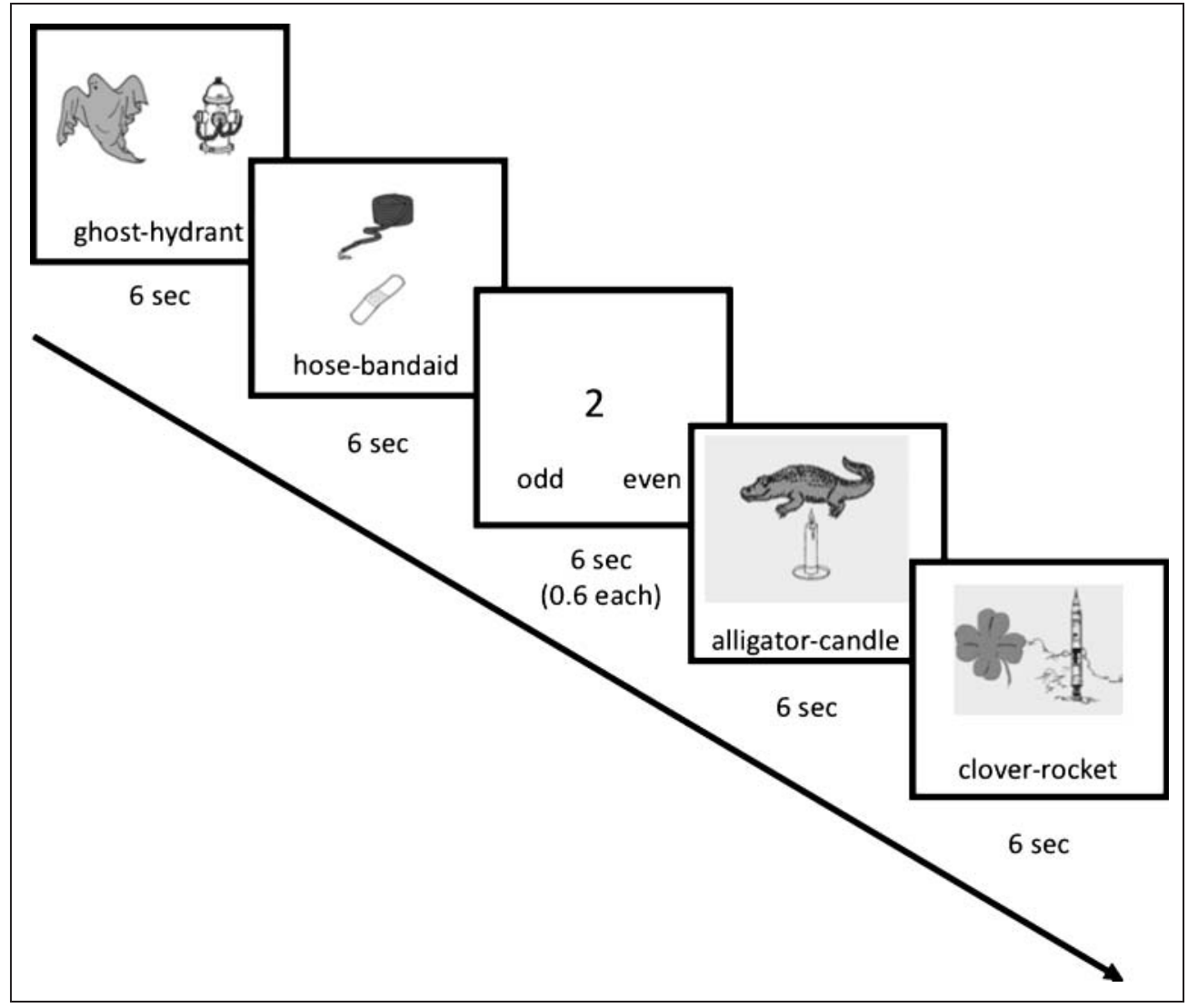


each group, and two-thirds were seen by both groups. For the nonoverlapping pairs, these items served as lures for the other group during retrieval. Retrieval stimuli consisted of 150 words corresponding to the cue items from the study phase, as well as 78 unstudied lure words. Although cue items remained the same for the 10-min and 1-week recognition tests, unique lures were used at each time point.

While being scanned, participants viewed stimuli via magnet-compatible goggles placed directly over the eyes (Resonance Technology Co., Inc., Northridge, CA). All stimuli were presented on a Macintosh Powerbook G4 computer using the MATLAB Psychophysics Toolbox.

\section{Procedure}

Participants were scanned during intentional encoding of object pairs. They were instructed to imagine the objects within each pair interacting, and to remember the details of each display as best as they could for a memory test following the encoding phase. Ten minutes later, participants performed the first of two recognition tests in which they made a studied/new judgment followed by a remember/ know judgment for those items deemed as studied. This two-step procedure has been shown to reduce participants' tendency to use the remember/know judgment as a simple confidence decision (Eldridge, Sarfatti, \& Knowlton, 2002). Participants were told to give an "R" response when they remembered the moment during which they had studied the item, and to give a " $\mathrm{K}$ " response when they confidently recognized an item in the absence of recollection. To ensure that participants properly understood this distinction, they were asked to define remember and know in their own words and to offer an example of each before beginning the recognition test.

Following the first testing session, participants were asked to return to the laboratory in one week's time, but were not told the purpose of this visit. Upon their return, they performed a second remember/know recognition test, identical to the first except for order of stimuli and use of unique lures. Finally, following this second recognition test, participants completed a posttest evaluating their memories for details of the encoding episode. Participants were presented with all 150 cue words from the encoding phase, and were prompted to indicate, via forced choice, the color of the cue object and the orientation of the object pair, and via recall, the object with which each cue item was paired. Completion of the posttest was self-paced (average duration $=32 \mathrm{~min}$ ); however, if participants had not completed the test within $45 \mathrm{~min}$, they were instructed to fill in the remaining items by guessing.

\section{Trial Distribution and Baseline Task}

Object pairs were distributed over three encoding scans, each consisting of 50 pairs presented for $6 \mathrm{sec}$, interspersed with 17 baseline trials, also lasting $6 \mathrm{sec}$. The order of encoding and baseline trials was determined using a genetic algorithm (Wager \& Nichols, 2003), such that amplitude differences between conditions were maximized.

Because participants may spontaneously engage in mnemonic processing during low-level fixation tasks, there is often more activity associated with fixation than a nonmnemonic baseline task. For this reason, the baseline task used in the present study was the odd/even digit task, which is shown to minimize hippocampal activity in comparison to passive fixation (Stark \& Squire, 2001). During baseline trials, participants saw a series of single digits on the screen for $600 \mathrm{msec}$ each, and were asked to determine if the digit was odd or even. A practice run of the odd/even task was administered prior to scanning to ensure that participants were able to perform the task given the rapid presentation rate.

\section{Data Acquisition and Preprocessing}

Structural and functional imaging was performed using a 3-T Siemens Allegra scanner. Structural images included (1) a sagittal localizer to identify the long axis of the hippocampus, (2) high-resolution T2 hippocampal images perpendicular to the long axis of the hippocampus ( $\mathrm{TR}=4 \mathrm{sec}, \mathrm{TE}=$ $105 \mathrm{msec}, 18$ slices, voxel size $0.4 \times 0.4 \times 3 \mathrm{~mm}, 20 \mathrm{~cm}$ FOV) for subsequent segmentation, (3) high-resolution gradient EPI sequences coplanar with the functional images ( $\mathrm{TR}=$ $5 \mathrm{sec}, \mathrm{TE}=66 \mathrm{msec}, 18$ slices, voxel size $1.6 \times 1.6 \times 3 \mathrm{~mm}$, $20 \mathrm{~cm}$ FOV) to aid alignment of the high-resolution structural images with the functional images, and (4) an MP-RAGE ( $\mathrm{TR}=2.3 \mathrm{sec}, \mathrm{TE}=2.93 \mathrm{msec})$ for future volumetric analyses. Functional imaging of the MTL was conducted with high-resolution, gradient-echo EPI sequences, consisting of 18 slices perpendicular to the long axis of the hippocampus $(\mathrm{TR}=3 \mathrm{sec}, \mathrm{TE}=39 \mathrm{msec}$, voxel size $1.6 \times$ $1.6 \times 3 \mathrm{~mm}, 20 \mathrm{~cm}$ FOV).

Preprocessing was performed using the FSL toolbox (www.fmrib.ox.ac.uk/fsl). Skulls were stripped using the Brain Extraction Tool (Smith, 2002), and functional images were realigned using McFLIRT to compensate for small head movement (Jenkinson, Bannister, Brady, \& Smith, 2002). For participants with translational motion over $1 \mathrm{~mm}$, images were de-noised using MELODIC (Beckmann \& Smith, 2004); however, no subjects showed motion greater than $2 \mathrm{~mm}$. Data were filtered with a high-pass cutoff of $75 \mathrm{sec}$, and were smoothed to only $2 \mathrm{~mm}$ given the high-resolution nature of the images.

\section{Response Classification}

Participants' responses from each of the two retrieval tests were classified as either remember $(\mathrm{R})$, know $(\mathrm{K})$, miss (M), correct rejection, or false alarm. For the purposes of back-sorting encoding trials, only $\mathrm{R}, \mathrm{K}$, and $\mathrm{M}$ trials were considered for further analysis. Because the same cue items were used for both retrieval sessions, we were able to track participants' memory across the 1-week delay. This procedure enabled us to conduct a subsequent 
Table 1. Overall Response Conditions According to Performance across the 10-min and 1-week Delay

\begin{tabular}{lllr}
\hline 10-min Delay & 1-week Delay & \multicolumn{1}{c}{ Response Condition } & Average No. of Trials \\
\hline R: Recollected & R: Recollected & RR: Consistently Recollected & $27.1(13-50)$ \\
R: Recollected & K: Familiar & RK: Transiently Recollected & $31.01(15-47)$ \\
K: Familiar & K: Familiar & KK: Consistently Familiar & $18.31(5-34)$ \\
M: Missed & M: Missed & MM: Consistently Missed & $15.91(5-50)$ \\
\hline R: Recollected & R: Recollected & RM: Recollected then Missed & $18.8(7-31)$ \\
K: Familiar & M: Missed & KR: Familiar then Recollected & 5.0 (1-12) \\
K: Familiar & R: Recollected & KM: Familiar then Missed & 11.5 (2-24) \\
M: Missed & K: Familiar & MR: Missed then Recollected & 2.6 (0-8) \\
M: Missed & MK: Missed then Familiar & $11.6(4-22)$ \\
\hline
\end{tabular}

Average number of trials per condition noted in the rightmost column (range noted in parentheses).

Response conditions included in our imaging analyses fall above the dividing line, those not included fall below it.

memory analysis by comparing activity related to items consistently recognized across both delays with that of items consistently forgotten across both delays. Subsequent memory analyses were performed on the following conditions of interest: consistently recollected (RR), initially recollected but later familiar (RK), consistently famil$\operatorname{iar}(\mathrm{KK})$, and consistently missed (MM) (Table 1). Although participant responses also led to an additional condition in which items were recognized across both delays (KR; initially familiar but later recollected), an insufficient number of trials existed to warrant inclusion in our imaging analyses. Finally, conditions in which performance was neither consistently successful nor unsuccessful were not included in our

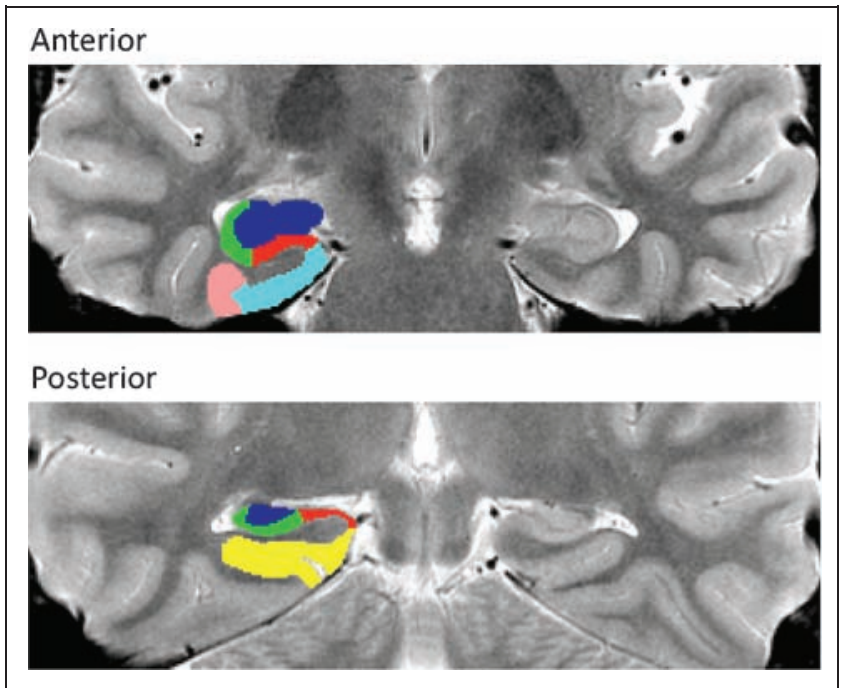

Figure 2. Anterior and posterior ROIs in a representative participant. Regions are delineated in the left hemisphere only so as to allow viewing of contralateral anatomy. Pink = perirhinal cortex; light blue = entorhinal cortex; yellow $=$ parahippocampal cortex; red $=$ subiculum; green $=\mathrm{CA} 1$; dark blue $=$ combined areas CA 2, 3, and the dentate gyrus (CA23/DG). analyses (RM, KM, MR, MK; see Table 1 for a breakdown of trial numbers across all conditions).

\section{Regions of Interest and Time-course Analyses}

Anatomical ROIs were created for each individual participant (Figure 2). Anatomical landmarks that were visible on each participant's high-resolution structural scan were used to delineate subregional boundaries. In the hippocampal formation, ROIs were created for the CA1 field, the CA $2 / 3$ fields and dentate gyrus, and the subiculum. The CA2 $/ 3$ fields and the dentate gyrus were not separable and, therefore, were collapsed into a single ROI (CA23/DG). Cortical ROIs surrounding the hippocampus included ERC, PRC, and PHC. All boundaries were defined using an anatomical atlas in the coronal plane (Duvernoy, 2005) and the specifications delineated in Amaral and Insausti (1990) as well as those adopted by structural studies of MTL subfields (Pruessner et al., 2000, 2002; Insausti et al., 1998). Furthermore, because susceptibility artifacts may be seen in the anterior regions of the MTL during EPI sequences, areas which were not visible in the functional scans were not included in the ROI analysis (Zeineh, Engel, \& Bookheimer, 2000). All ROIs were prepared by the same observer (V. A. C.) to maintain consistency.

Group time courses were created using the summary statistics approach to the mixed effects model (Mumford \& Nichols, 2006). First, time courses for each response type were extracted from each ROI in each participant using customized MATLAB routines employing a Finite Impulse Response (FIR) model. The design matrix in this model contained entries for each response condition, with a duration of $6 \mathrm{sec}$ across all conditions; baseline trials were left unmodeled. We estimated the underlying hemodynamic response for each trial type by averaging the signal across all voxels within an ROI at 3-sec bins beginning 6 sec prior to stimulus onset and ending $21 \mathrm{sec}$ after stimulus onset. 
The FIR model used was closely related to the selective averaging method of Dale and Buckner (1997) under the assumption of uncorrelated noise. For a given response type and participant, weighted least squares then was used to combine activation across the three encoding runs. Finally, time courses across all participants were averaged to create a group time course for each ROI.

To assess whether these time courses revealed differential contributions of MTL subregions to memory formation, we tested for a four-way interaction (via repeated measures ANOVA) between (1) hemisphere, (2) ROI, (3) response type, and (4) bin within the time course (bins included 0-18 sec). As reported in detail in the Results section, a significant three-way interaction was found between hemisphere, ROI, and response type; however, the four-way interaction involving the time course bin was not significant. Thus, we focused the remainder of our statistical analyses on differences in peak amplitude of the time courses. The peak was chosen by creating a grand average across all ROIs and all response types, and fell within the 6-sec bin. Investigations within each ROI were then conducted as planned comparisons of peak amplitude using paired, two-tailed $t$ tests $(\alpha=.05)$.

\section{RESULTS}

\section{Behavioral Results}

Participants accurately identified studied items during both retrieval tests. The hit rate for the 10-min test was $76.83 \pm$ $2.76 \%$ (standard error of the mean), with a false alarm rate of $16.45 \pm 3.88 \%$. The overall hit rate for the 1 -week test was $65.44 \pm 2.97 \%$, with a false alarm rate of $28.74 \pm 4.19 \%$. As expected, the 1-week delay caused some memories to fade; sorting hits into $\mathrm{R}$ and $\mathrm{K}$ responses, results show that participants' $\mathrm{R}$ rates decreased over the week delay, whereas $\mathrm{K}$ rates increased (see Figure 3 ). In both tests, $\mathrm{R}$ responses were more accurate than $\mathrm{K}$ responses in that false alarm

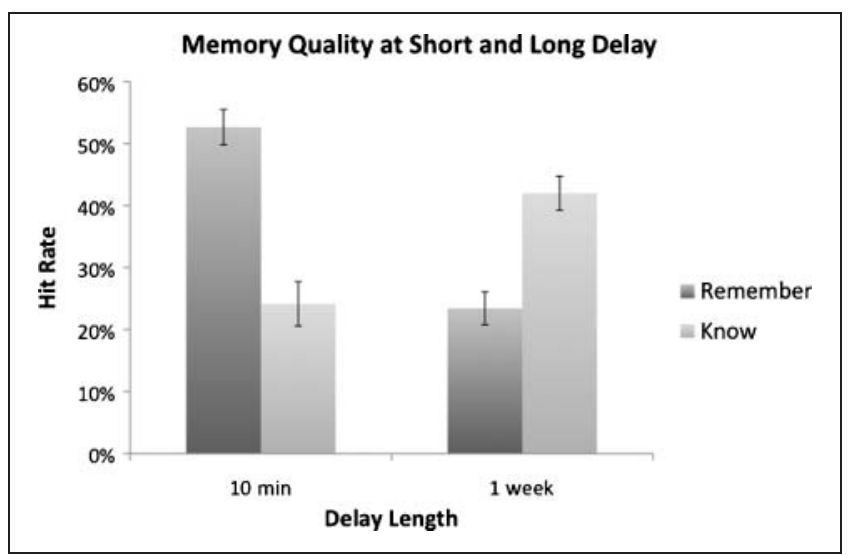

Figure 3. Behavioral results from the short (10-min) and long (1-week) delay recognition tests. Over time, the proportion of remember responses decreased, whereas know responses increased.

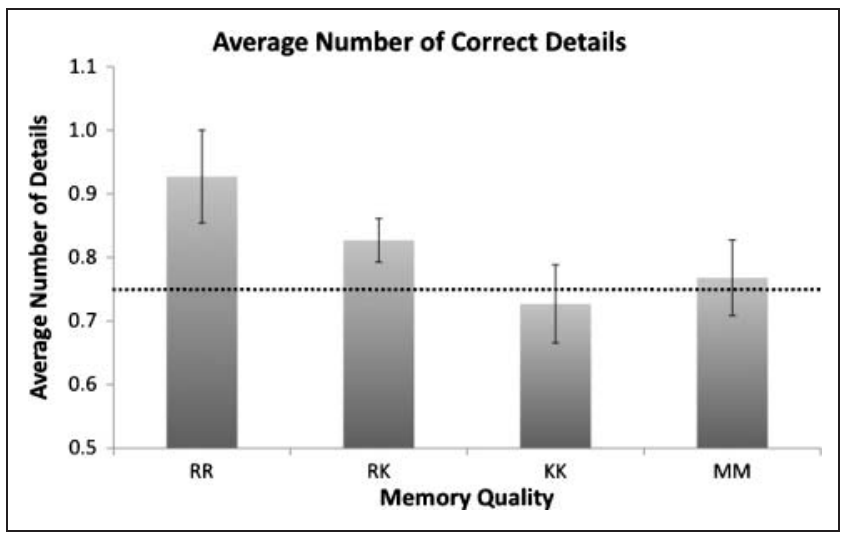

Figure 4. Behavioral results from the posttest conducted after the 1-week delay recognition test. Bars indicate average number of correct details associated with each response type (chance performance: 0.75 details) $. \mathrm{RR}=$ consistently recollected; $\mathrm{RK}=$ transiently recollected; $\mathrm{KK}=$ consistently familiar; $\mathrm{MM}=$ consistently forgotten .

rates were lower for $\mathrm{R}$ than for $\mathrm{K}$ items [10 min: $t(11)=$ 4.179, $p<.01 ; 1$ week: $t(11)=6.527, p<.0001]$. False alarm rates for $\mathrm{K}$ items were significantly higher after the long delay than after the short delay $(p<.01)$, but were not different across delays for $\mathrm{R}$ items.

Conditions of interest arising from behavioral performance across both retrieval tests are described in Table 1, along with a breakdown of trial numbers across conditions. Note that imaging analyses were inclusive of only RR, RK, $\mathrm{KK}$, and MM trials as described in the Methods section. Posttest results revealed differences in the number of details retrieved depending on response condition. KK items were more likely to co-occur with the retrieval of zero correct details than RR items $[t(11)=2.479, p<.05]$. KK items also showed fewer average details recalled than RR items $[t(11)=2.363, p<.05]$. Finally, the average number of details retrieved for KK and MM items was not significantly different than that predicted by chance $(p s>.7)$. The average number of correctly remembered details for each condition is shown in Figure 4.

\section{fMRI Results}

Analyses of fMRI data revealed distinct patterns of activity across subfields as a function of memory durability. A 2 (hemisphere) $\times 6($ ROI $) \times 4$ (response type) $\times 7$ (bin of time course) repeated measures ANOVA revealed significant main effects for ROI $[F(5,55)=4.288, p<.01]$ and bin of time course $[F(6,66)=23.012, p<.001]$. Additionally, a significant three-way interaction was found among hemisphere, ROI, and response type $[F(15,165)=$ $1.790, p<.05$ ], suggesting that the relationship among response types differed across individual ROIs. To further explore how these relationships differed, we performed planned comparisons based on peak amplitude of the time course in each ROI.

Four regions displayed significant subsequent memory effects, defined as greater activity for items later retrieved 
successfully than for items later forgotten: left CA23/DG, left PRC, and left and right PHC. In left CA23/DG, only consistently recollected items (RR) were associated with greater activity than consistently missed items $[t(11)=$ $2.201, p<.05]$; neither transiently recollected (RK; $p>$ .2) nor consistently familiar items (KK; $p<.5)$ differed from MM items (Figure 5). Left PRC showed a similar pattern: Only items that were consistently recollected (RR) showed greater activity than those that were consistently missed $[t(11)=2.726, p<.05]$; neither transiently recollected (RK; $p>$.4) nor consistently familiar items (KK; $p<.6$ ) differed from MM items (Figure 5). Activation patterns in right CA23/DG and right PRC revealed no significant effects.

Bilateral PHC displayed a different pattern of results, in which a subsequent memory effect was found for both consistently (RR) and transiently recollected (RK) items (Figure 5). Specifically, activity in left PHC associated with RR items was greater than that for MM items $[t(11)=$ $2.962, p<.05]$, as was activity associated with RK items $[t(11)=3.472, p<.01]$. A similar pattern of activity was found in right PHC, such that both RR and RK items were associated with greater than activity than MM items [RR: $t(11)=2.379, p<.05$; RK: $t(11)=2.375, p<.05]$. Consistently familiar items (KK) did not differ from MM items in either left or right PHC ( $p s>.5)$.

In those regions displaying a subsequent memory effect, we directly assessed involvement of each region in supporting subsequent durable versus transient recollection. Specifically, we compared activity associated with consistently recollected items (RR) with activity related to transiently recollected (RK) items, and further examined how each of these related to consistently familiar items (KK). Again, a similar pattern was found in left CA23/DG and left PRC, which differed from bilateral PHC. Left CA23/DG showed a trend toward significance for RR greater than RK items $[t(11)=1.801, p<.1]$, and in left PRC, RR items were associated with significantly greater activity than RK items $[t(11)=2.567, p<.05]$. Furthermore, in left PRC, activity for RR items was greater than that for KK items $[t(11)=2.754, p<.05]$, but activity for RK items was not significantly greater than that for KK items $(p>.7)$. Bilateral PHC, on the other hand, did not differentiate between consistently (RR) and transiently recollected (RK) items (left PHC, $p>.7$; right PHC, $p>$.3). In left PHC, there were trends for both RR and RK items to show greater activity than $\mathrm{KK}$ items $(p s<.1)$, and in right PHC, activity for RR items significantly differed from activity for KK items $[t(11)=$ $2.754, p<.05]$.

\section{DISCUSSION}

The present results demonstrate that activity in the MTL during encoding is associated with the durability of memory over time. Furthermore, these data suggest that subfields within the MTL contribute differentially to the formation of memories whose vividness endures and those that eventually fade to familiarity. Subsequent memory analyses revealed two patterns of results. First, in left CA23/DG and left PRC, encoding activity was associated with future memory success only if participants experienced recollection at both the 10-min and 1-week delay (consistently recollected). If participants' memories were initially accompanied by recollection yet later faded to familiarity (transiently recollected), activity in left CA23/DG and left PRC was not reliably enhanced above levels seen for consistently missed items. Similarly, items judged as consistently familiar did not engage these regions to a greater degree than consistently missed items. Second, in bilateral PHC, successful encoding activity was associated with both durable and transient recollection, such that activity for both response types was greater than that for consistently missed items, regardless of the presence or absence of recollection at the 1-week delay. As seen in CA23/DG and PRC, consistently familiar items were not associated with greater activity in bilateral PHC than consistently missed items.

To directly examine the role that encoding activity in these regions played in subsequently supporting durable versus transient recollection, we compared encoding activity for items consistently recollected across time with those that were initially recollected but later faded to familiarity. Encoding activity in left PRC differentiated between items that subsequently were recollected across both delays, and those that were transiently recollected. Left CA23/DG showed a similar pattern, although the comparison only reached trend levels of significance. Encoding activity in bilateral PHC, on the other hand, did not differentiate between consistently and transiently recollected items. Thus, although activity in each of these regions was associated with short-delay recollective success, activity in PHC did not discriminate between subsequent maintenance and loss of recollection after the 1-week delay.

\section{Functional Heterogeneity in the MTL}

\section{Hippocampus}

Our results in CA23/DG are in agreement with a large body of standard-resolution findings demonstrating an association between hippocampal encoding activity and

Figure 5. Subsequent memory analyses. Time courses represent percent signal change from baseline. Bar graphs display the peak amplitude of each trial type from which the peak amplitude of consistently missed (MM) items has been subtracted. Asterisks above bars indicate conditions demonstrating significantly greater activity than that associated with MM items. Additionally, in left PRC, a significant difference in the magnitude of the subsequent memory effect was found between items later consistently recollected (RR) and those transiently recollected (RK). RR: consistently recollected, RK: transiently recollected, KK: consistently familiar, MM: consistently forgotten. 


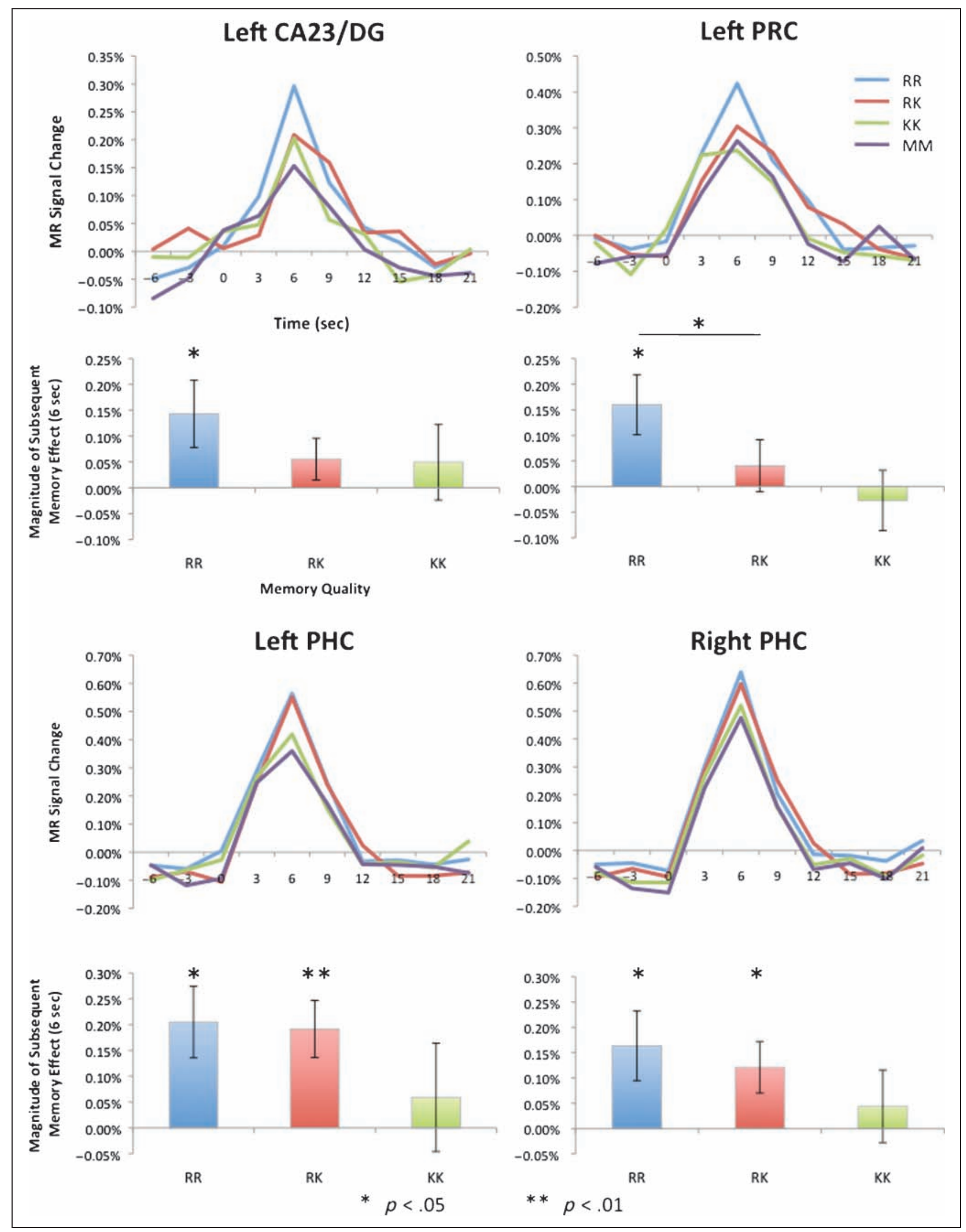


subsequent measures of recollection (for reviews, see Diana et al., 2007; Eichenbaum et al., 2007; Davachi, 2006). The current data extend these findings in two ways: (1) using highresolution imaging of the MTL, we were able to identify a specific subfield within the hippocampal formation that contributed most strongly to subsequent recollection, and (2) given our study design, we were able to show that activity in this region was correlated with subsequent recollection only if recollection endured over a week-long delay.

The involvement of CA23/DG, in particular, is supported by several recent high-resolution investigations of the MTL. Eldridge, Engel, Zeineh, Bookheimer, and Knowlton (2005) found that encoding activity in this region was associated with successful recollection $24 \mathrm{hr}$ after study. Furthermore, findings from Bakker, Kirwan, Miller, and Stark (2008) demonstrate that activity within CA23/DG differentiates between true stimulus repetitions and presentations of similar lure stimuli, suggesting a role of this region in the formation of distinct, nonoverlapping memory traces. With such a role in mind, it may be that CA23/DG is most active for consistently recollected items because activity in this region supports the formation of unique memories which are not likely to lose their distinctiveness over time. Findings from rodent work (Haberman, Lee, Colantuoni, Koh, \& Gallagher, 2008; Leutgeb, Leutgeb, Moser, \& Moser, 2007; Leutgeb, Leutgeb, Treves, Moser, \& Moser, 2004) and neural network models of MTL function (Rolls \& Kesner, 2006; O'Reilly \& McClelland, 1994; Treves \& Rolls, 1994) also suggest that the CA3 and DG regions are particularly well suited to form unique, detailed memories.

Our results also are consistent with the interpretation that hippocampal encoding activity is positively correlated with subsequent memory strength over time. Such an interpretation assumes that memory varies along a continuum of strength that is the result of contributions from both recollection and familiarity (for a review, see Squire et al., 2007). In our interpretation of the current findings we remain agnostic as to whether, e.g., an item remembered across both time points reflects consistent recollection or consistently high memory strength, and we instead focus on the relationship between encoding activity and the durability of an episodic memory over time. To this end, encoding activity in CA23/DG is greatest when memory remains episodic across the 1-week delay, and does not differ between conditions in which memories fade from episodic to weak, are consistently weak, or were never successfully encoded.

\section{MTL Cortex}

Turning next to cortical areas surrounding the hippocampus, we formulated two alternative hypotheses based upon findings from the computational and neuroimaging literatures. Many computational models posit that MTL cortices do not engage in encoding processes supportive of subsequent episodic recollection and instead support familiarity (e.g., Norman \& O'Reilly, 2003; O'Reilly \& Rudy, 2001). Accordingly, we hypothesized that although these regions would support successful memory formation, encoding activity would not differ according to the subsequent durability of recollection. A different consensus has emerged from the neuroimaging literature such that the posterior (PHC) and anterior (PRC) aspects of the parahippocampal gyrus are thought to encode different aspects of an experience (Diana et al., 2007; Eichenbaum et al., 2007; Davachi, 2006), with PHC more consistently implicated in encoding information that is capable of supporting recollection than PRC (Diana et al., 2007; Eichenbaum et al., 2007). To this end, we hypothesized that encoding activity in PHC would be greatest for items subsequently recollected across both delays, as was predicted for the hippocampus. Given discrepancies in the literature regarding the degree to which PRC engages in encoding processes capable of supporting subsequent recollection, we did not make specific predictions regarding the relationship between encoding activity in this region and the durability of recollection.

With respect to PHC, the current results fit well with our hypotheses based upon computational models, given that encoding activity in this region was associated with successful recognition across both delays but did not differ according to durability of recollection. It should be noted, however, that our findings do not suggest that this region plays no role in encoding episodic information; rather, the data demonstrate that PHC plays a nonselective role in encoding new memories such that the level of encoding activity is not associated with the degree to which a memory will maintain or lose vividness over time. A different pattern of results was found in PRC, however. Critically, this region demonstrated a subsequent memory effect only when items subsequently were recollected across both time points. Furthermore, PRC encoding activity was able to differentiate between items that subsequently were recollected across both delays, and those that were transiently recollected. Thus, data from PRC are not in agreement with predictions made by computational models regarding cortical encoding processes that are supportive of subsequent familiarity only.

In the imaging literature, findings from many subsequent memory studies point to a role for PRC in forming item-only or familiarity-based memories (e.g., Ranganath et al., 2004; Davachi, Mitchell, \& Wagner, 2003). However, recent research suggests that this region may be capable of encoding information in support of subsequent recollection under certain conditions, namely, encoding associations between items, or between items and their features processed as a unitized configuration (Haskins et al., 2008; Staresina \& Davachi, 2006, 2008; Tendolkar et al., 2007). Thus, the enhanced activity seen in this region during encoding may have contributed to future durable recollection in two ways: First, increased activity may reflect stronger encoding of the line drawings of each object - that is, memory for the items themselves. Second, increased activity may reflect stronger encoding of the color with which each cue item was associated. When memory is tested using cue words, those items for which a strong unified color-object 
representation had been formed may be more likely to be consistently recollected. As with the hippocampus, findings in PRC also fit with a memory strength interpretation. Thus, regardless of whether remember and know are thought to reflect qualitatively different memory states or different components of memory strength our findings suggest that enhanced encoding activity in PRC is associated with memories that will maintain their episodic character over time, whereas memories that lose this vividness are associated with significantly less PRC activity during encoding.

Taken together, activity patterns in hippocampal and MTL cortical subfields suggest that a simple recollection/ familiarity distinction between the hippocampus and parahippocampal gyrus does not sufficiently characterize MTL function. Rather, CA23/DG and PRC appear to play a more selective role in encoding memories subsequently maintaining their episodic character over time than PHC, whose activity reflected successful encoding regardless of the durability of a memory's vividness. Given the high-resolution nature of our functional images, we were unable to collect data outside the MTL; thus, we could not assess whether encoding activity in extra-MTL regions also was associated with the durability of subsequent recollection. In a comparable whole-brain study, however, Uncapher and Rugg (2005) found regions in the inferior frontal gyrus that were active during encoding of memories that persisted over a 48-hr delay. Future study of such regions using whole-brain imaging will be required to reveal areas outside the MTL associated with the encoding of memories that endure over time.

\section{Evaluation of Memory Durability}

Although the current study design was specifically chosen to allow for repeated testing of the same item across time such that memory durability could be tracked, using this paradigm may introduce a potential confound. It is possible that testing items at the 10-min delay made memory for the item more accessible at the 1-week test, thus weakening potential correlations between variability in encoding activity and subsequent durability of recollection. Although we could not control for this directly, we did attempt to reduce this possibility by using only cue words, and not the original pictures, during each recognition test. Furthermore, as participants made each remember/know judgment, they specifically were instructed to base their decision on whether or not they remembered the moment they originally saw the item, and were encouraged to try to remember details of how the object looked or with which item it was paired in making this decision.

Described in detail in Viskontas, Carr, Engel, and Knowlton (2009), high-resolution imaging data also were collected during the 10-min and 1-week recognition tests. Of interest to the current study is the degree to which subfield activity levels differed during the 10 -min retrieval test according to memory durability. In the event that recollecting a given item during this initial test influenced MTL activity, such that this item was more likely to be recollected again 1 week later, one would expect greater activity for consistently than transiently recollected items. However, an examination of activation levels in CA23/DG, PRC, and PHC during the 10-min test for recollected items revealed no significant differences between those items that would be recollected again at the 1 -week test and those that would not $(p s>1)$. Thus, it appears that activation during the intermediate test was not strongly associated with recollection on the final test. Additionally, our behavioral data show that participants very rarely exhibited memory quality at the 1-week test that was superior to that seen during the 10-min test (e.g., initially missed but later familiar, initially familiar but later recollected); trial numbers were, in fact, too low to warrant inclusion in our imaging analyses.

Finally, although it is likely that postencoding factors during the 10-min recognition test influenced subsequent performance on the 1-week test, significant differences in activation, nevertheless, were obtained during initial encoding for items that later were consistently or transiently recollected. Thus, neural activation during study appears to be an important factor in determining subsequent memory durability.

\section{Conclusions}

In conclusion, the present results demonstrate that the degree to which the MTL is active during encoding is associated with the durability of episodic recollection across a 1-week delay. Using high-resolution imaging techniques, we were able to demonstrate that activity in PRC and a specific subfield of the hippocampus, CA23/DG, reflected successful encoding only when items were consistently recollected across both delays. Furthermore, encoding activity in PRC for consistently recollected items was significantly greater than that for transiently recollected items. To the best of our knowledge, our findings reveal, for the first time, that encoding activity in the MTL differentiates between items that will maintain associated episodic content, and items for which recollection will rapidly fade. Thus, strength of MTL encoding activity appears to be an important factor in contributing to the degree to which our memories endure over time.

Reprint requests should be sent to Valerie A. Carr, Stanford University, Jordan Hall, Bldg 420, Mail Code 2130, Stanford, CA 94305, or via e-mail: valerie.carr@stanford.edu.

\section{REFERENCES}

Aggleton, J. P., \& Brown, M. W. (1999). Episodic memory, amnesia, and the hippocampal-anterior thalamic axis. Behavioral and Brain Sciences, 22, 425-444; discussion 444-489.

Amaral, D. G., \& Insausti, R. (1990). The hippocampal formation. San Diego, CA: Academic Press.

Bakker, A., Kirwan, C. B., Miller, M., \& Stark, C. E. (2008). Pattern separation in the human hippocampal CA3 and dentate gyrus. Science, 319, 1640-1642.

Beckmann, C. F., \& Smith, S. M. (2004). Probabilistic independent component analysis for functional magnetic resonance imaging. IEEE Transactions in Medical Imaging, 23, 137-152. 
Brewer, J. B., Zhao, Z., Desmond, J. E., Glover, G. H., \& Gabrieli, J. D. (1998). Making memories: Brain activity that predicts how well visual experience will be remembered. Science, 281, 1185-1187.

Brown, M. W., \& Aggleton, J. P. (2001). Recognition memory: What are the roles of the perirhinal cortex and hippocampus? Nature Reviews Neuroscience, 2, 51-61.

Dale, A. M., \& Buckner, R. L. (1997). Selective averaging of rapidly presented individual trials using fMRI. Human Brain Mapping, 5, 329-340.

Davachi, L. (2006). Item, context and relational episodic encoding in humans. Current Opinion in Neurobiology, 16, 693-700.

Davachi, L., Mitchell, J. P., \& Wagner, A. D. (2003). Multiple routes to memory: Distinct medial temporal lobe processes build item and source memories. Proceedings of the National Academy of Sciences, U.S.A., 100, 2157-2162.

Diana, R. A., Yonelinas, A. P., \& Ranganath, C. (2007). Imaging recollection and familiarity in the medial temporal lobe: A threecomponent model. Trends in Cognitive Sciences, 11, 379-386.

Duvernoy, H. (2005). The human hippocampus: Functional anatomy, vascularization and serial sections with MRI (3rd ed.). New York: Springer.

Eichenbaum, H., Yonelinas, A. P., \& Ranganath, C. (2007). The medial temporal lobe and recognition memory. Annual Review of Neuroscience, 30, 123-152.

Eldridge, L. L., Engel, S. A., Zeineh, M. M., Bookheimer, S. Y., \& Knowlton, B. J. (2005). A dissociation of encoding and retrieval processes in the human hippocampus. Journal of Neuroscience, 25, 3280-3286.

Eldridge, L. L., Sarfatti, S., \& Knowlton, B. J. (2002). The effect of testing procedure on remember-know judgments. Psychonomic Bulletin \& Review, 9, 139-145.

Fernandez, G., Weyerts, H., Schrader-Bolsche, M., Tendolkar, I., Smid, H. G., Tempelmann, C., et al. (1998). Successful verbal encoding into episodic memory engages the posterior hippocampus: A parametrically analyzed functional magnetic resonance imaging study. Journal of Neuroscience, 18, 1841-1847.

Haberman, R. P., Lee, H. J., Colantuoni, C., Koh, M. T., \& Gallagher, M. (2008). Rapid encoding of new information alters the profile of plasticity-related mRNA transcripts in the hippocampal CA3 region. Proceedings of the National Academy of Sciences, U.S.A., 105, 10601-10606.

Haskins, A. L., Yonelinas, A. P., Quamme, J. R., \& Ranganath, C. (2008). Perirhinal cortex supports encoding and familiaritybased recognition of novel associations. Neuron, 59, 554-560.

Insausti, R., Juottonen, K., Soininen, H., Insausti, A. M., Partanen, K., Vainio, P., et al. (1998). MR volumetric analysis of the human entorhinal, perirhinal, and temporopolar cortices. AJNR American Journal of Neuroradiology, 19, 659-671.

Jenkinson, M., Bannister, P., Brady, M., \& Smith, S. (2002). Improved optimization for the robust and accurate linear registration and motion correction of brain images. Neuroimage, 17, 825-841.

Leutgeb, J. K., Leutgeb, S., Moser, M. B., \& Moser, E. I. (2007). Pattern separation in the dentate gyrus and CA3 of the hippocampus. Science, 315, 961-966.

Leutgeb, S., Leutgeb, J. K., Treves, A., Moser, M. B., \& Moser, E. I. (2004). Distinct ensemble codes in hippocampal areas CA3 and CA1. Science, 305, 1295-1298.

Mumford, J. A., \& Nichols, T. (2006). Modeling and inference of multisubject fMRI data. IEEE Engineering in Medicine and Biology Magazine, 25, 42-51.

Norman, K. A., \& O'Reilly, R. C. (2003). Modeling hippocampal and neocortical contributions to recognition memory: A complementary-learning-systems approach. Psychological Review, 110, 611-646.

O’Reilly, R. C., \& McClelland, J. L. (1994). Hippocampal conjunctive encoding, storage, and recall: Avoiding a trade-off. Hippocampus, 4, 661-682.

O’Reilly, R. C., \& Rudy, J. W. (2001). Conjunctive representations in learning and memory: Principles of cortical and hippocampal function. Psychological Review, 108, 311-345.

Pruessner, J. C., Kohler, S., Crane, J., Pruessner, M., Lord, C. Byrne, A., et al. (2002). Volumetry of temporopolar, perirhinal, entorhinal and parahippocampal cortex from high-resolution MR images: Considering the variability of the collateral sulcus. Cerebral Cortex, 12, 1342-1353.

Pruessner, J. C., Li, L. M., Serles, W., Pruessner, M., Collins, D. L., Kabani, N., et al. (2000). Volumetry of hippocampus and amygdala with high-resolution MRI and three-dimensional analysis software: Minimizing the discrepancies between laboratories. Cerebral Cortex, 10, 433-442.

Ranganath, C., Yonelinas, A. P., Cohen, M. X., Dy, C. J., Tom, S. M., \& D'Esposito, M. (2004). Dissociable correlates of recollection and familiarity within the medial temporal lobes. Neuropsychologia, 42, 2-13.

Rolls, E. T., \& Kesner, R. P. (2006). A computational theory of hippocampal function, and empirical tests of the theory. Progress in Neurobiology, 79, 1-48.

Smith, S. M. (2002). Fast robust automated brain extraction. Human Brain Mapping, 17, 143-155.

Snodgrass, J. G., \& Vanderwart, M. (1980). A standardized set of 260 pictures: Norms for name agreement, image agreement, familiarity, and visual complexity. Journal of Experimental Psychology: Human Learning \& Memory, 6, 174-215.

Squire, L. R., Stark, C. E., \& Clark, R. E. (2004). The medial temporal lobe. Annual Review of Neuroscience, 27, 279-306.

Squire, L. R., Wixted, J. T., \& Clark, R. E. (2007). Recognition memory and the medial temporal lobe: A new perspective. Nature Reviews Neuroscience, 8, 872-883.

Staresina, B. P., \& Davachi, L. (2006). Differential encoding mechanisms for subsequent associative recognition and free recall. Journal of Neuroscience, 26, 9162-9172.

Staresina, B. P., \& Davachi, L. (2008). Selective and shared contributions of the hippocampus and perirhinal cortex to episodic item and associative encoding. Journal of Cognitive Neuroscience, 20, 1478-1489.

Stark, C. E., \& Squire, L. R. (2001). When zero is not zero: The problem of ambiguous baseline conditions in fMRI. Proceedings of the National Academy of Sciences, U.S.A., 98, 12760-12766.

Tendolkar, I., Arnold, J., Petersson, K. M., Weis, S., Anke, B.-D., van Eijndhoven, P., et al. (2007). Probing the neural correlates of associative memory formation: A parametrically analyzed eventrelated functional MRI study. Brain Research, 1142, 159-168.

Treves, A., \& Rolls, E. T. (1994). Computational analysis of the role of the hippocampus in memory. Hippocampus, 4, 374-391.

Tulving, E. (1985). Memory and consciousness. Canadian Psychology, 26, 1-12.

Uncapher, M. R., \& Rugg, M. D. (2005). Encoding and the durability of episodic memory: A functional magnetic resonance imaging study. Journal of Neuroscience, 25, 7260-7267.

Viskontas, I. V., Carr, V. A., Engel, S. A., \& Knowlton, B. J. (2009). The neural correlates of recollection: Hippocampal activation declines as episodic memory fades. Hippocampus, 19, 265-272.

Wager, T. D., \& Nichols, T. E. (2003). Optimization of experimental design in fMRI: A general framework using a genetic algorithm. Neuroimage, 18, 293-309.

Wagner, A. D., Schacter, D. L., Rotte, M., Koutstaal, W., Maril, A., Dale, A. M., et al. (1998). Building memories: Remembering and forgetting of verbal experiences as predicted by brain activity. Science, 281, 1188-1191.

Zeineh, M. M., Engel, S. A., \& Bookheimer, S. Y. (2000). Application of cortical unfolding techniques to functional MRI of the human hippocampal region. Neuroimage, 11, 668-683. 